\title{
Knockdown of JARID2 inhibits the viability and migration of placenta trophoblast cells in preeclampsia
}

\author{
YATAO JIANG, YUQING CHEN and YANPING CHEN \\ Department of Obstetrics and Gynecology, Fujian Provincial Hospital, Fuzhou, Fujian 350001, P.R. China
}

Received August 22, 2016; Accepted May 18, 2017

DOI: $10.3892 / \mathrm{mmr} .2017 .7011$

\begin{abstract}
Protein Jumonji (JARID2) is a member of the Jumonji family of proteins and has been demonstrated to regulate cell proliferation and invasion. However, little is known about the role of JARID2 in the metastasis of placenta trophoblast cells. In the present study, the effect and the underlying molecular mechanism of JARID2 on trophoblast cell viability and invasion was investigated. The expression of JARID2 in placental tissues was analyzed by reverse transcription-quantitative polymerase chain reaction and western blotting. HTR8/SVneo cells were transfected with si-JARID2 or scramble for $24 \mathrm{~h}$. Cell viability, migration and invasion in HTR8/SVneo cells were then evaluated. The expression levels of matrix metallopeptidase 2 (MMP2), MMP9, phosphorylated phosphatidylinositol 3-kinase (p-PI3K), PI3K, phosphorylated AKT serine/threonine kinase 1 (p-Akt) and Akt in HTR8/SVneo cells were also detected using western blotting. The results of the present study demonstrated that JARID2 is underexpressed in human preeclamptic placentas. The knockdown of JARID2 significantly inhibited the viability, migration and invasion of HTR8/SVneo cells. In addition, the knockdown of JARID2 significantly decreased the levels of phosphorylated PI3K and Akt in HTR8/SVneo cells. The results of the present study demonstrated that JARID2 may serve a role in the progression of preeclampsia. The knockdown of JARID2 inhibited the viability and invasion of trophoblast cells in preeclampsia by suppressing the PI3K/Akt signaling pathway. Therefore, JARID2 may serve as a novel potential target for treating preeclampsia.
\end{abstract}

\section{Introduction}

Preeclampsia is a serious pregnancy-related syndrome that remains a leading cause of maternal and perinatal morbidity and

Correspondence to: Dr Yuqing Chen, Department of Obstetrics and Gynecology, Fujian Provincial Hospital, 134 Wusi Road, Fuzhou, Fujian 350001, P.R. China

E-mail: yuqing_chenfj@126.com

Key words: protein Jumonji, preeclampsia, migration, phosphatidylinositol 3-kinase/Rac- $\alpha$ serine/threonine protein kinase pathway mortality. It is characterized by hypertension and proteinuria, with symptoms usually arising after 20 weeks of gestation (1). Despite research into preeclampsia, the molecular mechanisms underlying the onset and progression of the syndrome remain to be completely elucidated (2-4). Trophoblast cells are able to proliferate, migrate and invade the pregnant uterus during normal placental development. However, trophoblasts fail to invade the uterus in preeclamptic placentas (5). Therefore, an improved understanding of the molecular mechanisms underlying trophoblast invasion is required.

Protein Jumonji (JARID2) is a member of the Jumonji family of proteins (6). Previous studies have demonstrated that JARID2 acts as an accessory component of Polycomb repressive complex 2 (PRC2), which regulates important gene expression patterns during fetal development (7-9). JARID2 is associated with the maintenance of pluripotency and differentiation of embryonic stem cells (10). It has previously been observed that JARID2 is involved in the development and progression of a number of types of tumor such as lung and colon cancer $(11,12)$. Recently, Lei et al $(13)$ reported that downregulation of JARID2 inhibits hepatocellular carcinoma cell migration, invasion and proliferation in vitro, and metastasis in vivo. However, little is known about the role of JARID2 in the metastasis of placenta trophoblast cells. In the present study, the effect and underlying molecular mechanism of JARID2 in trophoblast cell viability and invasion was examined. The expression of JARID2 in placental tissues was analyzed by performing reverse transcription-quantitative polymerase chain reaction and western blotting. HTR8/SVneo cells were transfected with si-JARID2 or scramble for $24 \mathrm{~h}$, then cell viability, migration and invasion were evaluated. The expression levels of matrix metallopeptidase 2 (MMP2), MMP9, phosphorylated phosphatidylinositol 3-kinase (p-PI3K), PI3K, phosphorylated AKT serine/threonine kinase (p-Akt) 1 and Akt were also detected in HTR8/SVneo cells using western blotting. It was demonstrated that the knockdown of JARID2 inhibited trophoblast cell viability and migration through inactivation of the Rac- $\alpha$ Akt signaling pathway.

\section{Materials and methods}

Tissue specimens. Fresh placental tissues at 26-28 weeks of gestation were obtained from 3 healthy pregnant women (age, $24.2 \pm 2.4$ years) and from 3 female patients with preeclampsia (age, 25.7 \pm 1.8 years) who were recruited from the Department 
of Obstetrics and Gynecology, Fujian Provincial Hospital (Fujian, China), between July 2015 and December 2015. The specimens were immediately snap-frozen, and stored in liquid nitrogen until use. The present study was conducted with the approval of the Ethics Committee of Fujian Provincial Hospital (Fuzhou, China), and informed consent was obtained from all patients.

Cell culture. The human trophoblast cell line HTR8/SVneo was obtained from the American Type Culture Collection (Manassas, VA, USA). The cells were maintained in Dulbecco's modified Eagle's medium (DMEM; Invitrogen; Thermo Fisher Scientific, Inc., Waltham, MA, USA) supplemented with $10 \%$ fetal bovine serum (FBS; Sigma-Aldrich; Merck KGaA, Darmstadt, Germany), $100 \mathrm{U} / \mathrm{ml}$ penicillin and $100 \mathrm{mg} / \mathrm{ml}$ streptomycin (Sigma-Aldrich; Merck KGaA) at $37^{\circ} \mathrm{C}$ in a humidified $5 \% \mathrm{CO}_{2} / 95 \%$ air atmosphere.

RNA interference and transfection. The small interfering RNA against JARID2 (siRNA-JARID2; 5'-AGGAAGAGG AGGAGGACAA-3') and control siRNA (scramble; 5'-GAG UGGGUCUGGGUCUUCCCGUAGA-3') were chemically synthesized by Shanghai GenePharma Co., Ltd. (Shanghai, China). HTR8/SVneo cells were transfected for $24 \mathrm{~h}$ with siRNA-JARID2 or scramble using Lipofectamine ${ }^{\circledR} 2000$ (Invitrogen; Thermo Fisher Scientific, Inc.), according to the manufacturer's protocol.

Reverse transcription-quantitative polymerase chain reaction $(R T-q P C R)$. Total RNA was extracted from frozen tissues or cells using TRIzol reagent (Invitrogen; Thermo Fisher Scientific, Inc.). cDNA was synthesized from the extracted RNA (5 $\mu \mathrm{g})$ using the EasyScript First-Strand cDNA Synthesis SuperMix kit (Invitrogen; Thermo Fisher Scientific, Inc.). qPCR analysis was performed using a 7500 Real-Time PCR System (Applied Biosystems; Thermo Fisher Scientific, Inc.) with Fast Start Universal SYBR Green Master (Roche Diagnostics, Basel, Switzerland). PCR amplification was performed using the following primers: JARID2, 5'-GACACCAAACCCAATCACCAC-3' (sense) and 5'-GTTCAACCTGCCACTGACCTT-3' (antisense); and $\beta$-actin, 5'-TTAGTTGCGTTACACCCTTTC-3' (sense) and 5'-ACCTTCACCGTTCCAGTTT-3' (antisense). The thermocycling conditions were as follows: $95^{\circ} \mathrm{C}$ for $4 \mathrm{~min}$, followed by 40 cycles of $95^{\circ} \mathrm{C}$ for $25 \mathrm{sec}, 55^{\circ} \mathrm{C}$ for $30 \mathrm{sec}$ and $72^{\circ} \mathrm{C}$ for $20 \mathrm{sec}$ with $2 \mathrm{sec}$ for plate reading, then melting curve analysis from 65 to $95^{\circ} \mathrm{C}$. Data were analyzed using the formula: $\mathrm{R}=2^{-[\Delta \mathrm{Cq} \text { sample }-\Delta \mathrm{Cq} \text { control }]}(14)$.

Western blotting. Total protein was extracted from frozen tissues or cells using radioimmunoprecipitation assay lysis buffer (Beyotime Institute of Biotechnology, Haimen, China). Protein content was determined using a bicinchoninic acid protein assay (Pierce; Thermo Fisher Scientific, Inc.). Proteins (30 $\mu \mathrm{g} /$ lane) were separated using SDS-PAGE on a $12 \%$ gel and transferred onto a nitrocellulose membrane (EMD Millipore, Billerica, MA, USA). Following blocking with $5 \%$ fat-free milk in PBS containing $0.1 \%$ (v/v) Tween-20 at room temperature for $1 \mathrm{~h}$, membranes were incubated with one of the following mouse primary antibodies: anti-JARID2
(1:3,000; cat. no. SAB2105079; Sigma; Merck KGaA), anti-MMP2 (1:2,000; cat. no. sc-13594), anti-MMP9 (1:3,000; cat. no. sc-21733), anti-PI3K (1:2,000; cat. no. sc-365290), anti-p-PI3K (1:2,500; cat. no. sc-293115), anti-Akt (1:3,000; cat. no. sc-5298), anti-p-Akt (1:2,500; cat. no. sc-52940) or anti-GAPDH (1:2,000; cat. no. sc-47724; all Santa Cruz Biotechnology, Inc., Dallas, TX, USA) at $4^{\circ} \mathrm{C}$ overnight. Membranes were subsequently incubated with goat anti-mouse horseradish peroxidase (HRP)-conjugated immunoglobulin (Ig)G (1:2,500; cat. no. sc-2005; Santa Cruz Biotechnology, Inc.) or goat anti-rabbit HRP-conjugated $\operatorname{IgG}(1: 3,000$; cat. no. sc-2004; Santa Cruz Biotechnology, Inc.) for $1 \mathrm{~h}$ at room temperature. The target protein was visualized using enhanced chemiluminescence (Pierce; Thermo Fisher Scientific, Inc.) and densitometry was performed using Gel-Pro Analyzer software version 4.0 (Media Cybernetics, Inc., Rockville, MD, USA).

Cell viability assay. Cell viability was measured using the Cell Counting Kit-8 assay (CCK-8; Dojindo Molecular Technologies, Inc. Kumamoto, Japan). HTR8/SVneo cells were plated at a density of $1 \times 10^{4}$ cells/well in 96-well plates and transfected with siRNA-JARID2 or scramble. Following incubation for $24 \mathrm{~h}$, the CCK-8 reagents were added and incubated with the cells for $1 \mathrm{~h}$. Subsequently, the absorbance at $450 \mathrm{~nm}$ was measured using an ELISA plate reader.

Cell invasion and migration assays. Cell invasion assays were performed using Transwell ${ }^{\mathrm{TM}}$ chambers (Costar; Corning Incorporated, Corning, NY, USA). Transfected HTR8/SVneo cells $\left(1 \times 10^{4}\right.$ cells/well $)$ were plated in the top chamber of the insert, which had been precoated with Matrigel (BD Biosciences, Franklin Lakes, NJ, USA; $8 \mu \mathrm{m}$ pore size), and $500 \mu \mathrm{l}$ DMEM containing 10\% FBS was added to the lower chamber. Following $24 \mathrm{~h}$ of incubation, cells transferred to the lower surface of the base membrane were stained with hematoxylin and eosin (Sigma-Aldrich; Merck KGaA), and counted per 4 high power fields under a light microscope (magnification, $\mathrm{x} 100$ ). The migration assay was performed by the same procedure, except that the inserts were not pre-coated with Matrigel.

Statistical analysis. Data are expressed as the mean \pm standard deviation. All experiments were repeated at least three times. Statistical significance was analyzed using one-way factorial analysis of variance followed by a Dunnett's post hoc test or Student's two-tailed t-test. $\mathrm{P}<0.05$ was considered to indicate a statistically significant difference.

\section{Results}

JARID2 is underexpressed in preeclamptic placentas. In order to investigate the role of JARID2 in preeclampsia, the expression levels of JARID2 in placental tissues were examined by RT-qPCR analysis and western blotting. As presented in Fig. 1A, JARID2 mRNA levels were significantly decreased in placentas obtained from preeclamptic patients compared with placentas from healthy subjects. Similarly, the results of the western blot analysis indicated that the protein expression of JARID2 was significantly decreased 
A

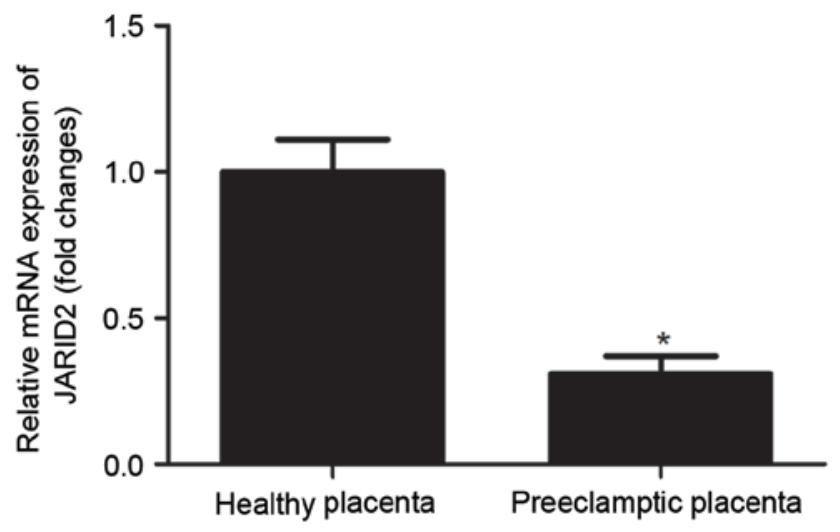

B
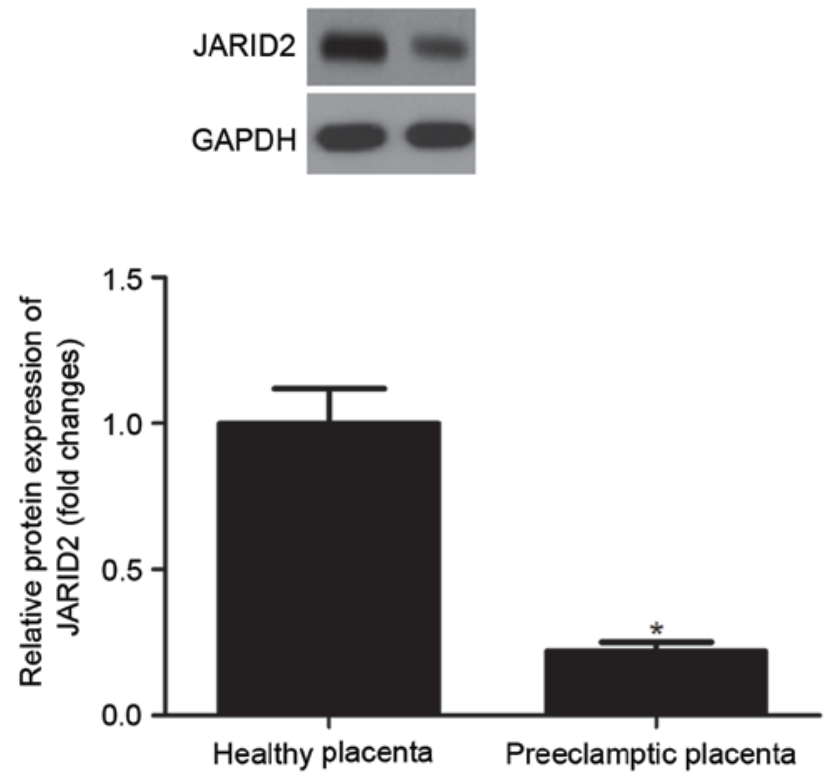

Figure 1. JARID2 is underexpressed in preeclamptic placentas. (A) The expression of JARID2 mRNA in placental tissues was analyzed by reverse transcription-quantitative polymerase chain reaction, with quantification relative to $\beta$-actin. (B) The expression of JARID2 protein in placental tissues was analyzed by western blotting, with quantification relative to GAPDH. Data are presented as the mean \pm standard deviation of three independent experiments. ${ }^{*} \mathrm{P}<0.05$ vs. healthy placenta. JARID2, protein Jumonji.

in preeclamptic placentas compared with the control group (Fig. 1B).

Knockdown of JARID2 inhibits HTR8/SVneo cell viability. In order to gain further insight into the effect of JARID2 on cell viability, JARID2 was downregulated using siRNA against JARID2 in HTR8/SVneo cells. As demonstrated in Fig. 2A and B, respectively, siRNA knockdown of JARID2 significantly decreased the expression of JARID2 at the mRNA and protein levels in HTR8/SVneo cells. In addition, the effects of si-JARID2 on HTR8/SVneo cell viability were examined. The results of the CCK-8 assay demonstrated that, compared with the scramble group, knockdown of JARID2 significantly inhibited the viability of HTR8/SVneo cells (Fig. 2C).
A

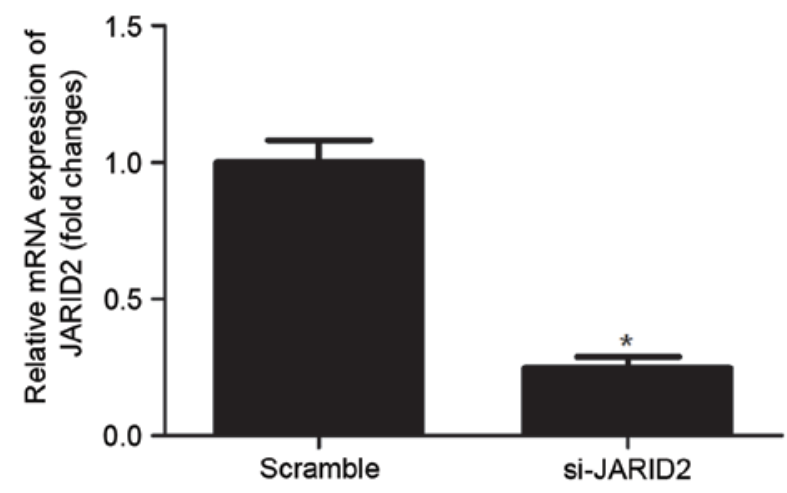

B
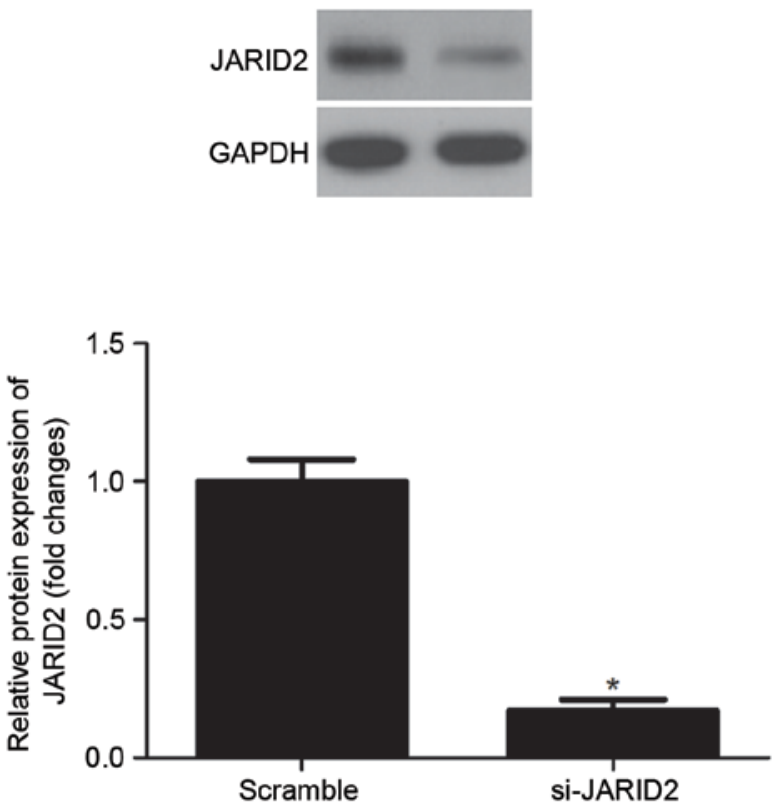

C

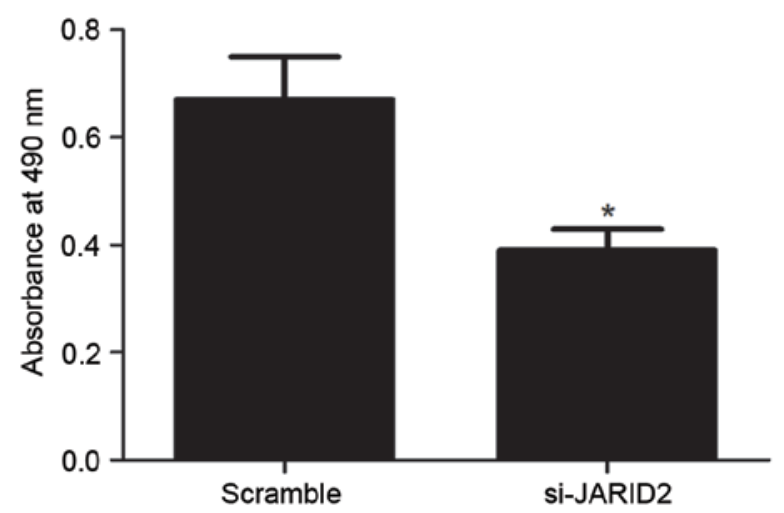

Figure 2. Knockdown of JARID2 reduces HTR8/SVneo cell viability. (A) mRNA and (B) protein expression of JARID2 was significantly decreased in HTR8/SVneo cells transfected with si-JARID2 compared with cells transfected with scramble. (C) Cell viability was assessed by Cell Counting Kit-8 assay. Data are presented as the mean \pm standard deviation of three independent experiments. ${ }^{*} \mathrm{P}<0.05$ vs. scramble. si, small interfering; JARID2, protein Jumonji. 
A

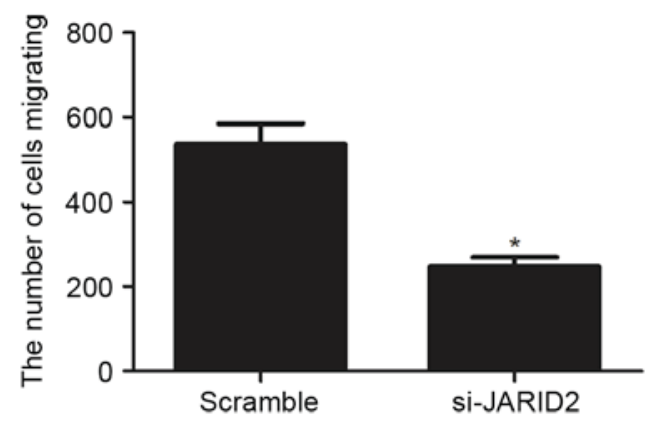

C
B
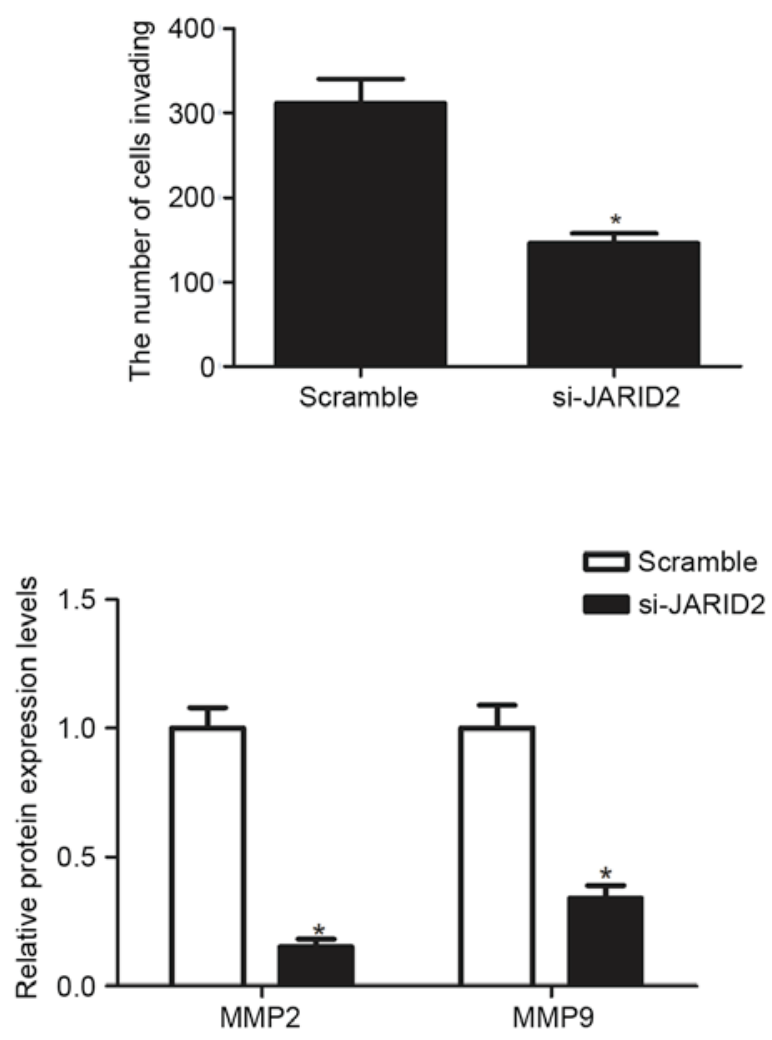

Figure 3. Knockdown of JARID2 inhibits the migration and invasion of HTR8/SVneo cells. HTR8/SVneo cells were transfected with si-JARID2 or scramble for $24 \mathrm{~h}$. (A) Cell migration was evaluated using the Transwell migration assay. (B) Cell invasion was determined using the Matrigel invasion assay. (C) Western blotting was used to detect the levels of MMP2 and MMP9 protein expression in transfected HTR8/SVneo cells. Data are presented as the mean \pm standard deviation of three independent experiments. ${ }^{*} \mathrm{P}<0.05$ vs. respective scramble. JARID2, protein Jumonji; si, small interfering; MMP, matrix metallopeptidase.

Knockdown of JARID2 inhibits the migration and invasion of HTR8/SVneo cells. The present study investigated the effects of JARID2 on HTR8/SVneo cell migration and invasion. The Transwell migration assay demonstrated that the knockdown of JARID2 significantly inhibited the migratory capacity of HTR8/SVneo cells, compared with the scramble group (Fig. 3A). The Matrigel assay demonstrated that the number of cells that passed through the Matrigel-coated membrane into the lower chamber was significantly decreased in si-JARID2-transfected cells compared with scramble-transfected cells (Fig. 3B). In addition, the present study analyzed the effect of JARID2 on MMP expression in HTR8/SVneo cells. The results of the western blot analysis demonstrated that knockdown of JARID2 significantly decreased the expression of MMP2 and MMP9 protein, compared with the scramble group (Fig. 3C).

Knockdown of JARID2 inhibits the activation of PI3K/Akt pathway in HTR8/SVneo cells. In order to gain insights into the downstream signaling pathways modulated by si-JARID2 in preeclampsia inhibition, the present study investigated the effect of si-JARID2 on the activation of the PI3K/Akt signaling pathway in HTR8/SVneo cells. As presented in Fig. 4, the knockdown of JARID2 significantly decreased the levels of phosphorylated PI3K and Akt in HTR8/SVneo cells, compared with the scramble group.

\section{Discussion}

Preeclampsia is a pregnancy-specific complication and is associated with insufficient extravillous trophoblast invasion. To the best of our knowledge, the present study is the first to investigate the expression and role of JARID2 in preeclampsia. The results of the present study demonstrated that JARID2 is underexpressed in human preeclamptic placentas. The knockdown of JARID2 significantly inhibited the viability and migration/invasion of HTR8/SVneo cells. Additionally, the knockdown of JARID2 reduced the activation of PI3K and Akt in HTR8/SVneo cells.

JARID2 has been observed to regulate the proliferation of a number of types of cells. Mejetta et al (10) confirmed that silencing JARID2 decreases the proliferation of rhabdomyosarcoma cells by inducing myogenic differentiation. By contrast, it has been reported that the knockdown of JARID2 promotes leukemia cell proliferation via acceleration of the G1/S transition (15). These previous findings suggest that JARID2 may serve promotive or inhibitory functions depending on the cell type or context. In the present study, it was observed that JARID2 is underexpressed in human preeclamptic placentas, and that the knockdown of JARID2 inhibited the viability of HTR8/SVneo cells. The present results suggested that JARID2 may act as a suppressor in the development and progression of preeclampsia. 
A

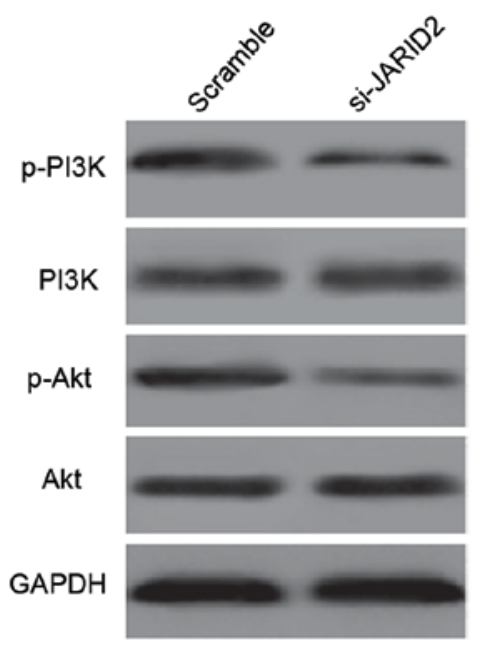

B

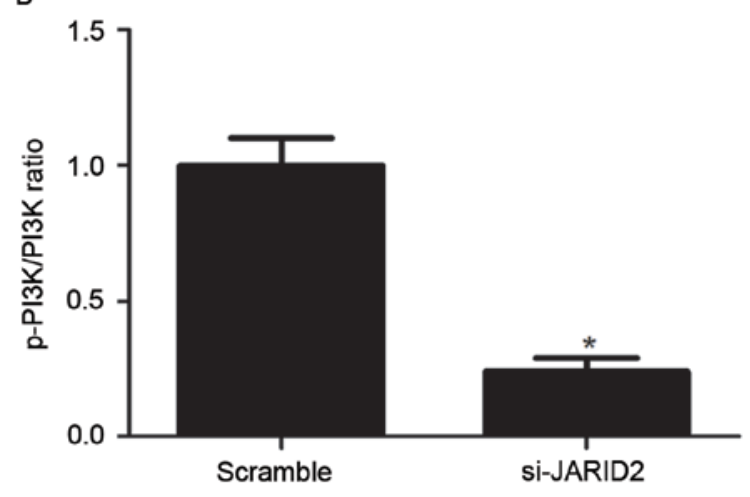

C

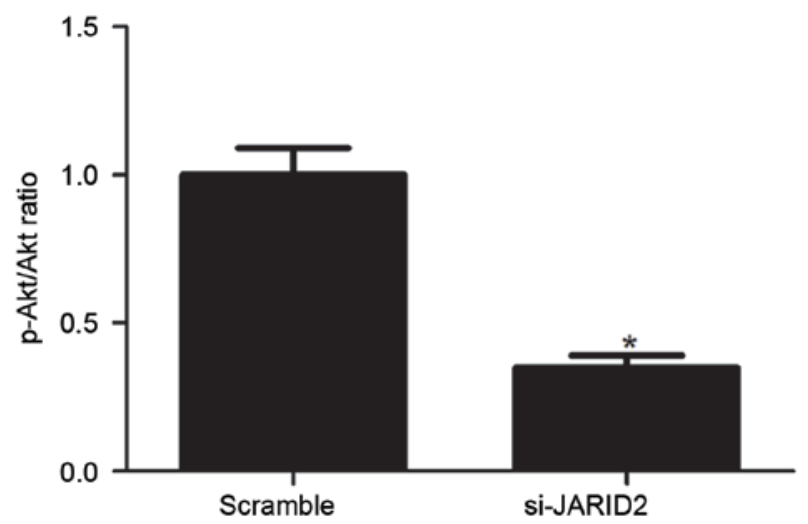

Figure 4. Knockdown of JARID2 inhibits the activation of the PI3K/Akt signaling pathway in HTR8/SVneo cells. HTR8/SVneo cells were transfected with si-JARID2 or scramble for $24 \mathrm{~h}$. (A) Western blotting was used to detect the protein expression levels of p-PI3K, PI3K, p-Akt and Akt in HTR8/SVneo cells, with quantification relative to GAPDH. PI3K and Akt activation was assessed by examination of relative levels of (B) p-PI3K/PI3K and $(\mathrm{C}) \mathrm{p}$-Akt/Akt. Data are presented as the mean \pm standard deviation of three independent experiments. ${ }^{*} \mathrm{P}<0.05$ vs. scramble. JARID2, protein Jumonji; si, small interfering; p, phosphorylated; PI3K, phosphatidylinositol 3-kinase; Akt, AKT serine/threonine kinase 1.

Previous studies have demonstrated that deficient migration and shallow invasion of trophoblasts may lead to preeclampsia $(16,17)$. JARID2 has been suggested to regulate tumor cell migration and invasion; a previous study revealed that knockdown of JARID2 markedly inhibits hepatocellular carcinoma cell migration and invasion (13). Consistent with the results of this previous study, in the present study, it was observed that the knockdown of JARID2 significantly inhibited the viability and migration/invasion of HTR8/SVneo cells. Additionally, MMPs serve important roles in cell migration and invasion by remodeling the extracellular matrix $(18,19)$. During early pregnancy, for embryo implantation and placentation, the invasion of human trophoblast cells depends on the secretion of MMPs, primarily MMP2 and MMP9 (20,21). In the present study, it was observed that the knockdown of JARID2 significantly inhibited the expression of MMP2 and MMP9 in HTR8/SVneo cells. The present study provides evidence to suggest that the knockdown of JARID2 may lead to decreased MMP2 and MMP9 expression, which may lead to insufficient trophoblast cell migration and invasion, thereby contributing to preeclampsia.

Previous studies have reported that the PI3K/Akt signaling pathway may serve important roles in regulating the proliferation, migration and invasion of trophoblast cells (22-24). Akt is a serine/threonine protein kinase, and the phosphorylation of Akt has been observed to be decreased in the preeclamptic placenta (25). In addition, activated Akt is able to promote cell motility and invasion (26,27). Therefore, the suppression of PI3K/Akt signaling may be a promising approach for treating preeclampsia. A recent study reported that JARID2 promotes the activation of Akt in hepatocellular carcinoma (13). In the present study, it was observed that the knockdown of JARID2 suppressed the levels of phosphorylated PI3K and Akt in HTR8/SVneo cells. The results of the present study suggested that the knockdown of JARID2 inhibited the viability and invasion of trophoblast cells in preeclampsia by suppressing the PI3K/Akt signaling pathway.

In conclusion, the results of the present study demonstrated that JARID2 may serve an important role in the progression of preeclampsia. The knockdown of JARID2 inhibited the viability and invasion of trophoblast cells in preeclampsia by suppressing the PI3K/Akt signaling pathway. Therefore, JARID2 may serve as a novel potential target for treating preeclampsia.

\section{References}

1. Backes CH, Markham K, Moorehead P, Cordero L, Nankervis CA and Giannone PJ: Maternal preeclampsia and neonatal outcomes. J Pregnancy 2011: 214365, 2011.

2. Dieplinger $\mathrm{H}$ : Method for diagnosing preeclampsia. Patent WO2013000992 A1. Filed June 28, 2012; issued January 3, 2013.

3. Bernardi F, Guolo F, Bortolin T, Petronilho F and Dal-Pizzol F: Oxidative stress and inflammatory markers in normal pregnancy and preeclampsia. J Obstet Gynaecol Res 34: 948-951, 2008.

4. Berzan E, Doyle R and Brown CM: Treatment of preeclampsia: Current approach and future perspectives. Curr Hypertens Rep 16: 473, 2014.

5. Lyall F: Mechanisms regulating cytotrophoblast invasion in normal pregnancy and pre-eclampsia. Aust N Z J Obstet Gynaecol 46: 266-273, 2006.

6. Kooistra SM and Helin K: Molecular mechanisms and potential functions of histone demethylases. Nat Rev Mol Cell Biol 13: 297-311, 2012.

7. Herz HM and Shilatifard A: The JARID2-PRC2 duality. Genes Dev 24: 857-861, 2010. 
8. Pasini D, Cloos PA, Walfridsson J, Olsson L, Bukowski JP, Johansen JV, Bak M, Tommerup N, Rappsilber J and Helin K: JARID2 regulates binding of the Polycomb repressive complex 2 to target genes in ES cells. Nature 464: 306-310, 2010.

9. Kinkel SA, Galeev R, Flensburg C, Keniry A, Breslin K, Gilan O, Lee S, Liu J, Chen K, Gearing LJ, et al: Jarid2 regulates hematopoietic stem cell function by acting with polycomb repressive complex 2. Blood 125: 1890-1900, 2015.

10. Mejetta S, Morey L, Pascual G, Kuebler B, Mysliwiec MR, Lee Y, Shiekhattar R, Di Croce L and Benitah SA: Jarid2 regulates mouse epidermal stem cell activation and differentiation. EMBO J 30: 3635-3646, 2011.

11. Tange S, Oktyabri D, Terashima M, Ishimura A and Suzuki T: JARID2 is involved in transforming growth factor-beta-induced epithelial-mesenchymal transition of lung and colon cancer cell lines. PLoS One 9: e115684, 2014.

12. Manceau G, Letouzé E, Guichard C, Didelot A, Cazes A, Corté H Fabre E, Pallier K, Imbeaud S, Le Pimpec-Barthes F, et al: Recurrent inactivating mutations of ARID2 in non-small cell lung carcinoma. Int J Cancer 132: 2217-2221, 2013.

13. Lei X, Xu JF, Chang RM, Fang F, Zuo CH and Yang LY: JARID2 promotes invasion and metastasis of hepatocellular carcinoma by facilitating epithelial-mesenchymal transition through PTEN/AKT signaling. Oncotarget 7: 40266-40284, 2016.

14. Livak KJ and Schmittgen TD: Analysis of relative gene expression data using real-tie quantitative PCR and the 2(-Delta Delta C(T)) Method. Methods 25: 402-408, 2001.

15. Su CL, Deng TR, Shang Z and Xiao Y: JARID2 inhibits leukemia cell proliferation by regulating CCND1 expression. Int J Hematol 102: 76-85, 2015.

16. Suga N, Sugimura M, Koshiishi T, Yorifuji T, Makino S and Takeda S: Heparin/heparan sulfate/CD44-v3 enhances cell migration in term placenta-derived immortalized human trophoblasts cells. Biol Reprod 86: 134, 1-8, 2012.

17. Zou Y, Jiang Z, Yu X, Sun M, Zhang Y, Zuo Q, Zhou J, Yang N, Han $\mathrm{P}, \mathrm{Ge} \mathrm{Z}$, et al: Upregulation of long noncoding RNA SPRY4-IT1 modulates proliferaiton, migraiton, apoptosis, and netword formation in trophoblast cell HTR-8SV/neo. PLoS One 8: e79598, 2013.
18. Kim YH, Kwon HJ and Kim DS: Matrix metalloproteinase 9 (MMP-9)-dependent processing of $\beta$ ig-h3 protein regulates cell migration, invasion, and adhesion. J Biol Chem 287: 38957-38969, 2012.

19. Chen Q, Jin M, Yang F, Zhu J, Xiao Q and Zhang L: Matrix Metalloproteinases: Inflammatory regulators of cell behaviors in vascular formation and remodeling. Mediators Inflamm 2013: 928315, 2013

20. Staun-Ram E, Goldman S, Gabarin D and Shalev E: Expression and importance of matrix metalloproteinase 2 and 9 (MMP-2 and -9) in human trophoblast invasion. Reprod Biol Endocrinol 2: 59, 2004.

21. Seval Y, Akkoyunlu G, Demir R and Asar M: Distribution patterns of metalloproteinase (MMP)-2 and-9 and their inhibitors (TIMP-1 and TIMP-2) in the human decidua during early pregnancy. Acta Histochem 106: 353-362, 2004

22. Zheng Q, Dai K, Cui X, Yu M, Yang X, Yan B, Liu S and Yan Q: Leukemia inhibitory factor promote trophoblast invasion via urokinase-type plasminogen activator receptor in preeclampsia. Biomed Pharmacother 80: 102-108, 2016.

23. Jia RZ, Ding GC, Gu CM, Huang T, Rui C, Wang YX and Lu Q: CDX2 enhances HTR-8/SVneo trophoblast cell invasion by altering the expression of matrix metalloproteinases. Cell Physiol Biochem 34: 628-636, 2014.

24. Liu X, Mu H, Luo X, Xiao X, Ding Y, Yin N, Deng Q and Qi H: Expression of Gadd45 $\alpha$ in human early placenta and its role in trophoblast invasion. Placenta 35: 370-377, 2014.

25. Cudmore MJ, Ahmad S, Sissaoui S, Ramma W, Ma B, Fujisawa T, Al-Ani B, Wang K, Cai M, Crispi F, et al: Loss of Akt activity increases circulating soluble endoglin release in preeclampsia: Identification of inter-dependency between Akt-1 and heme oxygenase-1. Eur Heart J 33: 1150-1158, 2012.

26. BuljZ, Duchi S, Bevilacqua A, Gherardi A, Dozza B, Piccinini F, Adalgisa Mariani G, Lucarelli E, Giannini S, Donati D and Marmiroli S: Protein kinase B/AKT isoform 2 drives migration of human mesenchymal stem cells. Int J Oncol 42: 118-126, 2013.

27. Yoeli-Lerner $M$ and Toker A: Akt/PKB signaling in cancer: $A$ function in cell motility and invasion. Cell Cycle 5: 603-605, 2006. 